\title{
Assessing the Elevation of Cardiac Biomarkers and the Severity of COVID-19 Infection: A Meta-analysis
}

\author{
Cucnhat P. Walker ${ }^{\mathrm{a}}$, Subrata Deb ${ }^{\mathrm{b}}$, Hua Ling ${ }^{\mathrm{c}}$, Zhe Wang ${ }^{\mathrm{d}}$ \\ aDepartment of Clinical and Administrative Sciences, College of Pharmacy, Larkin University, Miami, FL 33169, USA; \\ ${ }^{b}$ Department of Pharmaceutical Sciences, College of Pharmacy, Larkin University, Miami, FL 33169, USA; 'Department of \\ Pharmacy Practice, School of Pharmacy, Philadelphia College of Osteopathic Medicine, Suwanee, GA 30024, USA; \\ ${ }^{\mathrm{d} D e p a r t m e n t ~ o f ~ P h a r m a c y ~ P r a c t i c e, ~ T o u r o ~ C o l l e g e ~ o f ~ P h a r m a c y, ~ N e w ~ Y o r k, ~ N Y ~ 10027, ~ U S A ~}$
}

Corresponding author: Cucnhat P. Walker, Pharm.D., M.P.H, BCPS, Department of Clinical and Administrative Sciences, College of Pharmacy, Larkin University, 18301 North Miami Avenue, Suite 1, Miami, FL 33169; Tel: 305-760-7494; Email: ciciwalker2018@gmail.com

Received, October 12, 2020; Revised, October 16, 2020; October, October 17, 2020; Published, October 24, 2020

\begin{abstract}
Purpose: Since December 2019, coronavirus disease 2019 infection has become a global pandemic. The cases of Coronavirus Disease 2019 (COVID-19)-related acute cardiac injury with unknown pathophysiologic mechanism has become increasingly prevalent. However, it is not yet understood how the extent of cardiac injury differs with the intensity of viral infection. In the current study, we aimed to assess the association between elevated cardiac biomarkers and the severity of COVID-19 infection. Methods: A systematic literature search was performed across PubMed and Embase databases from December 1, 2019 to July 10, 2020, to identify studies that reported cardiac biomarkers of troponin (TnI) and creatine kinase-myocardial band (CK-MB) in patients with COVID-19. These studies compared non-severe patients with severe patients, or survivors with nonsurvivors or medical patients with critically ill patients. The data were extracted for TnI, CK-MB, N-terminalbrain natriuretic peptide (NT-BNP), D-dimer, and lactate dehydrogenase (LDH), C-reactive protein (CRP), and interleukin 6 (IL-6). Wherever possible, the data were pooled for meta-analysis (Review Manager, RevMan. version 5.3) with standard or weighted mean or median difference and corresponding $95 \%$ confidence intervals (95\% CI). Results: A total of 25 studies involving 5,626 patients were included in the present analysis. More severe COVID-19 infection was found to be associated with higher mean values of TnI $(-0.54[-0.72,-0.36])$ (ng/mL), CK-MB (-1.55 [-2.23, -0.88]) (ng/mL) and (-4.75 [-13.31, 3.82]) (units/L), NT-BNP (-815.7 [-1073.97, -557.42]) (pg/mL), D-dimer (-1.4 [-2.04, -0.77]) (mcg/mL), and LDH (-176.59 [-224.11, -129.06]) (units/L), as well as CRP (-64.03 [-68.88, -59.19]) (mg/L) and IL-6 (-22.59 [-29.39, -15.79]) (pg/mL). Conclusions: There is significant association between elevated cardiac biomarkers and the severity of COVID-19, which underlines the increased risk of acute cardiac injury with more severe viral infection. This highlights the need to understand the cardiac history among the COVID-19 patients during initial assessment and for monitoring.
\end{abstract}

\section{INTRODUCTION}

On March 11, 2020, the World Health Organization (WHO) designated Coronavirus Disease 2019 (COVID-19) as a global pandemic following the first reported human case in December 2019 (1). As of October 7, 2020, the WHO has reported over 35 million COVID-19 cases and more than 1 million deaths worldwide (2). In most patients, the clinical presentation of COVID-19 includes fever, cough, headache, and shortness of breath (3). However, inflammatory response to COVID-19 leads to systemic increased inflammatory markers and potential abnormal function of vital organ systems, including pulmonary and cardiovascular (4). COVID-19 enters pulmonary and cardiac cells through the angiotensin converting enzyme 2 (ACE2) protein as entry receptors, in addition to its invasion into the vasculature via the gastrointestinal tract (4). From there, it can cause direct damage to the cells or induce an inflammatory response by activation of cytotoxic T-lymphocytes. Additionally, patients with prior history of cardiac diseases may experience recurrent cardiovascular events due to 
atherosclerotic plaque rupture, catecholamine release, or demand ischemia $(3,5)$.

The cases of COVID-19-related acute cardiac injury have become increasingly prevalent. One of the most prominent types of cardiac injury is myocarditis. Myocarditis generally occurs due to inflammation of the myocardium without probable ischemic etiology. In the United States, Canada, and other developed countries, viral infection is the most common cause. Other common causes of myocarditis include bacteria (e.g., streptococcus, staphylococcus), toxins, and inflammatory disorders (e.g. sarcoidosis) $(3,6,7)$. While endomyocardial biopsy is the most accurate diagnostic test, it is rarely performed due to invasiveness and higher level of expertise required to interpret results (8). Other types of COVID-19-related cardiac injury include cytokine release syndrome, acute coronary syndrome, stress induced cardiomyopathy, and sepsis induced cardiomyopathy $(3,6,7)$. The primary goal of this meta-analysis is to investigate the association between cardiac biomarker elevations and the severity of COVID-19 infection.

\section{METHODS}

\section{Data Sources, Search Terms and Inclusion Criteria}

This meta-analysis was conducted based on the Preferred Reporting Items for Systematic Review and Meta-Analyses (PRISMA), and in compliance with the Meta-analysis Of Observational Studies in Epidemiology (MOOSE) checklist (9, 10). Electronic searches were performed for all clinical studies describing cardiac and inflammatory enzymes in patients infected with COVID-19 in PubMed and EMBASE databases through July 10, 2020. The searches were conducted using the following key words: "covid-19", "coronavirus disease 2019", "SARS-Cov-2", "severe acute respiratory syndrome coronavirus 2", "2019-nCOV", "2019 novel coronavirus", "coronavirus", "cardiac injury", "troponin", "arrhythmia". A manual search in the secondary sources such as references of initially identified articles, reviews, and commentaries was done to identify additional relevant studies. Duplications were screened by Rayyan (https://rayyan.qcri.org) and double-checked independently by two investigators (CPW, SD) before removal. These two investigators also independently assessed the search results for relevancy by titles, abstracts, and/or full texts in Rayyan. Conflicts were resolved via discussion and consensus. The studies were included based on the following inclusion criteria: 1) studies of adult patients infected with COVID-19, 2) studies included troponin I (TnI) and creatine kinasemyocardial band (CK-MB), 3) studies included a comparison group such as severe versus (vs.) nonsevere or mild vs. moderate vs. severe or critical vs. non-critical or survivors vs. non-survivors. The articles were restricted to English language. Studies that stratified patient populations based on the history of specific disease conditions (i.e., diabetes mellitus, obesity, stroke, ST-elevation myocardial infarction, chronic kidney disease, kidney transplantation) were excluded. The articles were assessed independently by the third investigator (HL) and differences were resolved via discussion and consensus among all investigators.

\section{Laboratory Markers}

The primary focus of this data analysis was to investigate the association between the elevations of cardiac biomarkers (TnI, CK-MB, N-terminal-brain natriuretic peptide (NT-BNP), D-dimer, and lactate dehydrogenase (LDH)) and the severity of COVID19 infection. The inflammatory biomarkers such as C-reactive protein (CRP) and interleukin-6 (IL-6) were also assessed to understand the potential mechanism behind cardiac injury with their significant elevations. These biomarkers were found to be the most common in all the included studies. If only the median and interquartile range (IQR25, IQR75) were reported, then it was assumed that the median was equal to the mean and that the standard deviation (SD) was (Q75-Q25)/1.35.

\section{Data Analysis}

Using Review Manager (RevMan. version 5.3. Copenhagen: The Nordic Cochrane Centre, The Cochrane Collaboration, 2014), the Binary Random Effects model with the DerSimonian-Laird method was applied to calculate mean differences of these laboratory parameters between non-severe group (mild and moderate) and severe group of COVID-19 infected patients. The $I^{2}$ statistic test was performed to assess $I^{2}$ of $<25 \%, 25-50 \%, 50-75 \%$, and $>75 \%$ indicating no, low, moderate, and high degree of inbetween study heterogeneity, respectively. The statistical significance was set at $95 \%$ confidence interval $(95 \% \mathrm{CI})$ and $\mathrm{p}$ value $<0.05$. Each laboratory parameter from the included studies were reported in different units, which were all converted into one common unit for the final analysis, with the only 
exception for CK-MB. There were six studies reported $\mathrm{CK}-\mathrm{MB}$ in $\mathrm{ng} / \mathrm{mL}$ and four studies in units/L; hence, two separate analyses were performed based on these two units for this biomarker.

\section{RESULTS}

\section{Study Characteristics}

As depicted in Figure 1, a total of twenty five studies involving 5,626 patients were included in our analysis $(11-33,43,44)$. Thirteen studies compared COVID-19 survivors with non-survivors, while the remainder compared non-severe patients with severe patients or medical patients with intensive care unit (ICU) patients. Overall, the mean or median age in these studies were in the range of 50s to 60s years of age, with the severe or non-survivor group being significantly older, except for Chen et al. study with age being insignificantly different (11). Various comorbidities were assessed and compared between two groups, except Medetalibeyoglu et al. and Zheng et al. studies which did not assess comorbidities of the subjects $(12,13)$. The comorbidities that were more significantly common in the severe groups included cardiovascular diseases (i.e., coronary artery disease, heart failure) in ten studies (14-24), hypertension in nine studies $(14,16-19,21,22,24$, $25)$, chronic respiratory diseases in nine studies (17, 19, 20, 23-28), diabetes mellitus in seven studies (11, $16,18,21,22,25,29)$, cerebrovascular diseases in five studies $(15,16,18,21,24)$, chronic kidney disease in four studies $(18,22-24)$, cancer in one study (25), and immunosuppression in one study (17). Four studies did not find significant association between the infection severity and the patients' comorbidities (30-33). Tanriverdi et al. study only found the overall significant association of all comorbidities combined with the infection severity (27). The most common initial symptoms reported were high fever, dry cough, and dyspnea. Less common symptoms were headache, dizziness, abdominal pain, diarrhea, and nausea-vomiting.

Only eight studies in our analysis described the active treatments for COVID-19 infection and empiric treatment for bacterial and/or fungal coinfections during hospitalization $(21,22,28,29,32$,
33, 38, 43). These treatments included antiviral agents (i.e., arbidol, oseltamivir, lopinavir-ritonavir, ribavirin, ganciclovir, interferon-alpha), antibiotics (i.e., fluoroquinolones, cephalosporins, imipenem or meropenem, linezolid, penicillins, azithromycin), antifungal agents, glucocorticoid (i.e., methylprednisone), unspecified corticosteroid, and immunoglobulin. Among these therapies, only antiviral therapies were found to make no significant difference in patient outcome between two groups in all these studies.

\section{Difference in Cardiac Markers and Disease Severity}

All laboratory parameters, both cardiac (TnI, CKMB, NT-BNP, D-dimer, and LDH) and inflammatory (CRP and IL-6) biomarkers, were found to have significant mean differences between the non-severe group and the severe group, except CK-MB (units/L) (Figures 2-9). Because CK-MB values were reported in $\mathrm{ng} / \mathrm{mL}$ in six studies and in units/L in four studies, two separate analyses were performed for each unit. The mean difference of CKMB in $\mathrm{ng} / \mathrm{mL}$ between the two groups was found to be significant at $-1.55,95 \% \mathrm{CI}[-2.23,-0.88]$ while it was not the case for the mean difference of CK-MB in units/L between the two groups $(-4.75,95 \%$ CI [$13.31,3.82]$. Heterogeneity of the studies were very high for all biomarkers with $I^{2}$ ranging from $79 \%$ to $98 \%$ (p < 0.1), except NT-BNP (units/L) with $I^{2}$ of $27 \%(\mathrm{p}=0.23)$.

\section{DISCUSSION}

This meta-analysis was aimed to compare the status of different cardiac and inflammatory markers with the severity of COVID-19. Our data demonstrated that levels of cardiac biomarkers (TnI, CK-MB, NTBNP, D-dimer, and LDH) as well as inflammatory markers (CRP and IL-6) were significantly elevated in patients with severe COVID-19 infection compared with non-severe infection. Severe COVID-19 cases tend to be older and have preexisting diseases, specifically cardiovascular diseases, hypertension, cerebrovascular disease, chronic pulmonary diseases, diabetes mellitus, cancer, and immunosuppression. 


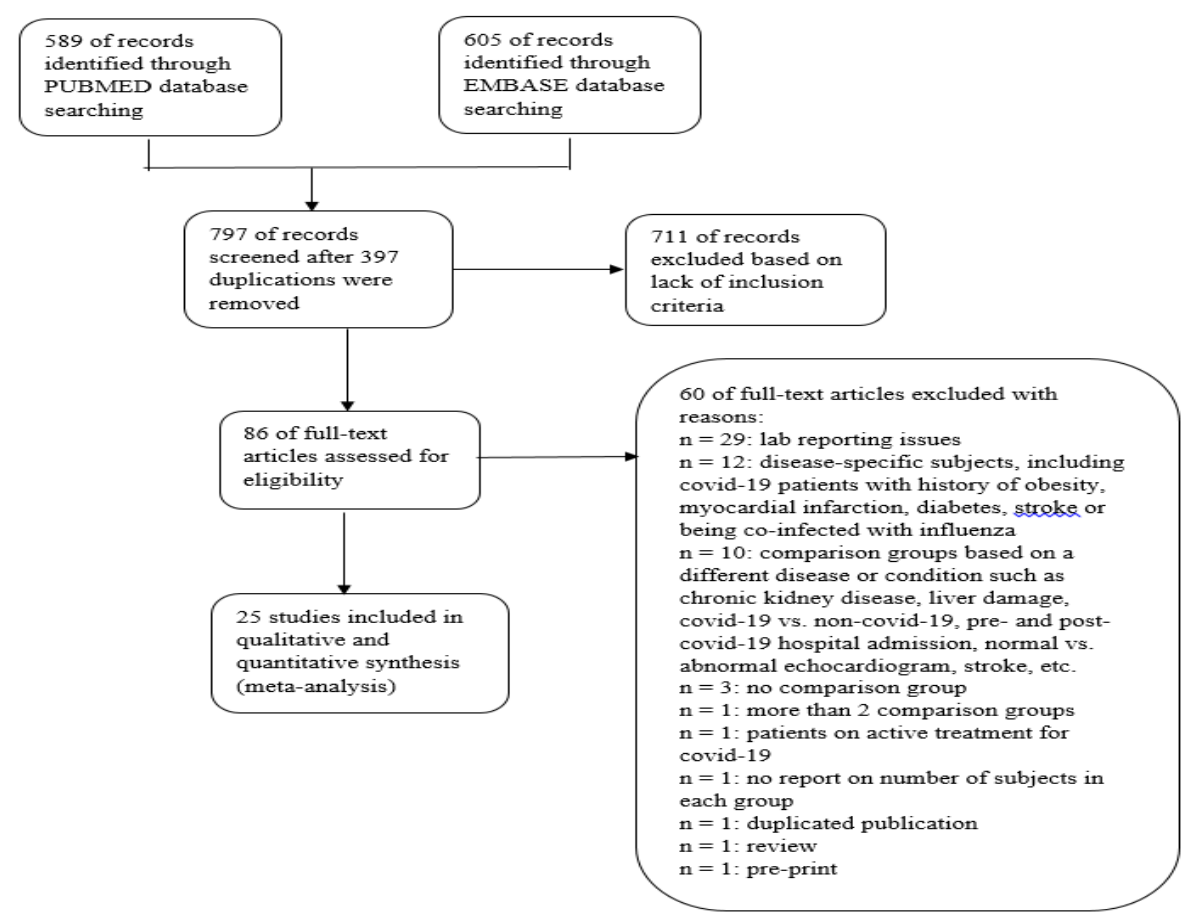

Figure 1. The PRISMA flow chart demonstrates systematic literature review, identification of studies, article screening, and study selection.

The findings from the current study are consistent with previously conducted meta-analyses on COVID-19 and cardiac injury. An earlier meta-analysis of 28 reports, which included published patient data from December 16, 2019 to February 20, 2020, found that cardiac injury biomarkers (TnI, CK-MB, NT-proBNP) were significantly higher in severely ill COVID-19 patients compared to non-severe cases and were also associated with pre-existing hypertension $(\mathrm{p}=0.03)$ (34). Mortality was higher in patients with acute cardiac injury compared to those without (summary risk ratio (RR) 3.85 [2.13, $6.96], \mathrm{p}<0.001)$ and in more severe cases compared to non-severe (summary RR 13.90 [7.32, 13.90], $\mathrm{p}<0.001$ ). This analysis did not find a significant difference in serum myoglobin levels between severe and non-severe cases. It was noted that this meta-analysis included publications in non-PubMed indexed journals.

A second meta-analysis of 13 reports of (search finalized on March 29, 2020) showed that cardiac injury was associated with higher mortality (RR 7.95 [5.12, 12.34], $\left.\mathrm{p}<0.001 ; I^{2}=65 \%, \mathrm{p}=0.009\right)$ and a higher need for ICU care (RR 7.94 [5.52, 34.52], p < 0.001; $I^{2}=0 \%$, $\mathrm{p}=0.38$ ) (35). The level of TnI was significantly higher in patients with acute cardiac injury (mean difference 10.38 $\left.\mathrm{pg} / \mathrm{mL}[4.44,16.32], \mathrm{p}=0.002, I^{2}=0 \%, \mathrm{p}=0.92\right)$. The most current meta-analysis at the time of our review analyzed 12 studies (published between December 20, 2019 and March 15, 2020) and confirmed the previous findings that mortality from COVID-19 infection was significantly associated with non-survivors and elevated cardiac biomarkers (TnI, LDH, and CK-MB) (36). Among the non-survivor patients, risk factors for cardiovascular disorders including male gender, age (>50 years), and comorbidities such as dyslipidemia, smoking, hypertension, and diabetes were more prevalent. These three meta-analyses analyzed reported data only from Chinese patients in various provinces in China, especially Wuhan. Our study is the first to include reports of patients from wider geographical regions (i.e., China, Korea, Turkey, Italy, Spain, Iran, the United States, Switzerland, England). Also, since we excluded the studies that classified patients based on pre-existing cardiac, liver or metabolic diseases, our findings more closely reflect the effect of COVID-19 on cardiac health in the infected patients based on the viral disease severity.

TnI and CK-MB are two major cardiac biomarkers that are affected by COVID-19 infection. Because TnI is critical in regulating the interplay between myosin and actin via calcium, different negative changes with the cardiac structure is responded with the elevation of TnI (37). From several studies with COVID-19 patients, it can be observed that these cardiac markers are elevated as a result of diverse mechanistic damage to the cardiomyocytes and eventually heart. Examples of potential mechanisms of cardiac injury from COVID-19 include damage to the microvascular structure, ischemia from low oxygen availability, immune response-related inflammatory syndrome, and direct viral infection of the heart (11, 37-40). From previous non-COVID-19 studies, it is known that inflammation can negatively affect 
cardiovascular health through actuating the reninangiotensin system (RAS) and thus disturbing the homeostasis of vasodilator and vasoconstrictor angiotensin peptides in the heart $(41,42)$. Since both TnI and CK-MB are structural cardiac proteins, the severity of COVID-19 infection will determine the intensity of changes in these cardiac-specific markers. Indeed, the studies included in the present meta-analysis indicate that these markers are significantly different between the nonsevere and severe patients. For example, the intensity of acute respiratory distress syndrome of the patients can determine the level of oxygen supply and ischemic injury to cardiomyocytes and eventually liberation of troponin into the general circulation (43). Similarly, CK-MB, which is generally located in the myocardium, can be released following cytokine storm-related hyperinflammatory situations or other putative mechanisms during COVID-19 infection (40). Inflammation of the myocardium mediated by COVID19-generated interleukins and tumor necrosis factor-a can lead to myocyte necrosis, loss of contractility, and eventually severe cardiomyopathy (43). In addition, angiotensin-converting enzyme 2 (ACE2), which is considered to be the main anchoring protein of SARSCoV-2, is abundantly expressed in the heart (11). This facilitates direct viral infection of the cardiac structures, leading to cardiac injury. Overall, the COVID-19 infection can influence the cardiac-specific biomarkers through direct or indirect mechanisms, leading to acute or longterm cardiac dysfunction. Since there is no medical standard of care available at this time, efforts should be made to lower the systemic inflammation in the COVID19 patients. Examples of potential agents to decrease proinflammatory cytokine effects include tocilizumab (anti-IL-6 receptor monoclonal antibody) and gevokizumab (anti-IL-1 $\beta$ monoclonal antibody) $(47,48)$.

The current study has several limitations. Firstly, there is a significant heterogeneity among the studies, which is as expected and in line with the current published literature. However, unlike previous systematic reviews and meta-analyses, we excluded non-PubMed indexed articles and pre-prints to ensure only peer-reviewed publications be included. Secondly, in some of the clinical studies we identified various reporting units for laboratory markers, which resulted in $10^{6}$ factors in difference upon unit conversion (i.e., 0.01 to 10,000). We speculate that possible errors might have occurred in reporting those laboratory marker units. In addition, CK-MB was reported in both $\mathrm{ng} / \mathrm{mL}$ and units/L, which was not possible to be converted to a common unit. Two separate analyses were performed for each unit, resulting in one being statistically significant and the other not. Thirdly, we chose TnI and $\mathrm{CK}-\mathrm{MB}$ as our primary cardiac enzymes as part of our inclusion criteria; hence, any studies without one or both of the markers were excluded. Though TnI and CK-MB are the clinically preferred diagnostic markers of cardiac health, there is also a possibility that we have excluded studies with other cardiac enzymes (i.e., proNT-BNP, Ddimer, myoglobin) that could be useful in assessing cardiac injury in COVID-19 patients. Fourthly, although only eight studies in our analysis reported COVID-19 treatments (i.e., antivirals, antibiotics, immunoglobulin, corticosteroids), they did not address the cardiovascular effects of these medications. Azithromycin was cited as part of the COVID-19 treatment in these studies but not hydroxychloroquine. There have been several studies, both observational studies and randomized control trials, demonstrating the risk of QT prolongation with hydroxychloroquine, chloroquine, and/or azithromycin $(49,50,51,52)$. On April 24, 2020, the US Food \& Drug Administration published a drug safety communication warning health care professionals and patients of the known risk of QT prolongation associated with hydroxychloroquine or chloroquine in combination with azithromycin, especially their increased use through the outpatient prescriptions for treating or preventing COVID19 (53). On the side note, in the comprehensive review by Kohan and colleagues, all the agents currently used to COVID-19 infection have direct or indirect antiinflammatory effects and/or direct or indirect interactions with ACE-2 protein. They suggested that the influence of inflammation on response to cardiovascular drugs, and the choice of medication are important considerations in COVID-19 treatment since the infection is associated with cardiovascular complication (54). Fifthly, Yang et al. reported 129 out of 136 patients in their retrospective study were treated with Chinese traditional medicines in addition to Western medicines (22). These herbal medicines were not specified; hence, it is unknown the extent of drug-herb interactions among these patients and whether these interactions had any adverse effects on the patients' overall clinical condition and acute cardiac injury specifically. This leads us to ponder if patients included in other studies in China were treated with this combination regimen and were not reported in their publications. Finally, we did not analyze the significance of pre-existing comorbidities and mortality associated with acute cardiac injury in COVID-19 infection.

\section{CONCLUSIONS}

There is significant association between elevated cardiac biomarkers and the severity of COVID-19, which underscores the increased risk of acute cardiac injury with more severe viral infection. This calls for attention of the healthcare team to understand the cardiac history of COVID-19 patients during the initial assessment and monitor their cardiac function during the progression of COVID-19.

CONFLICT OF INTEREST:

All authors have no conflict of interests to disclose. 


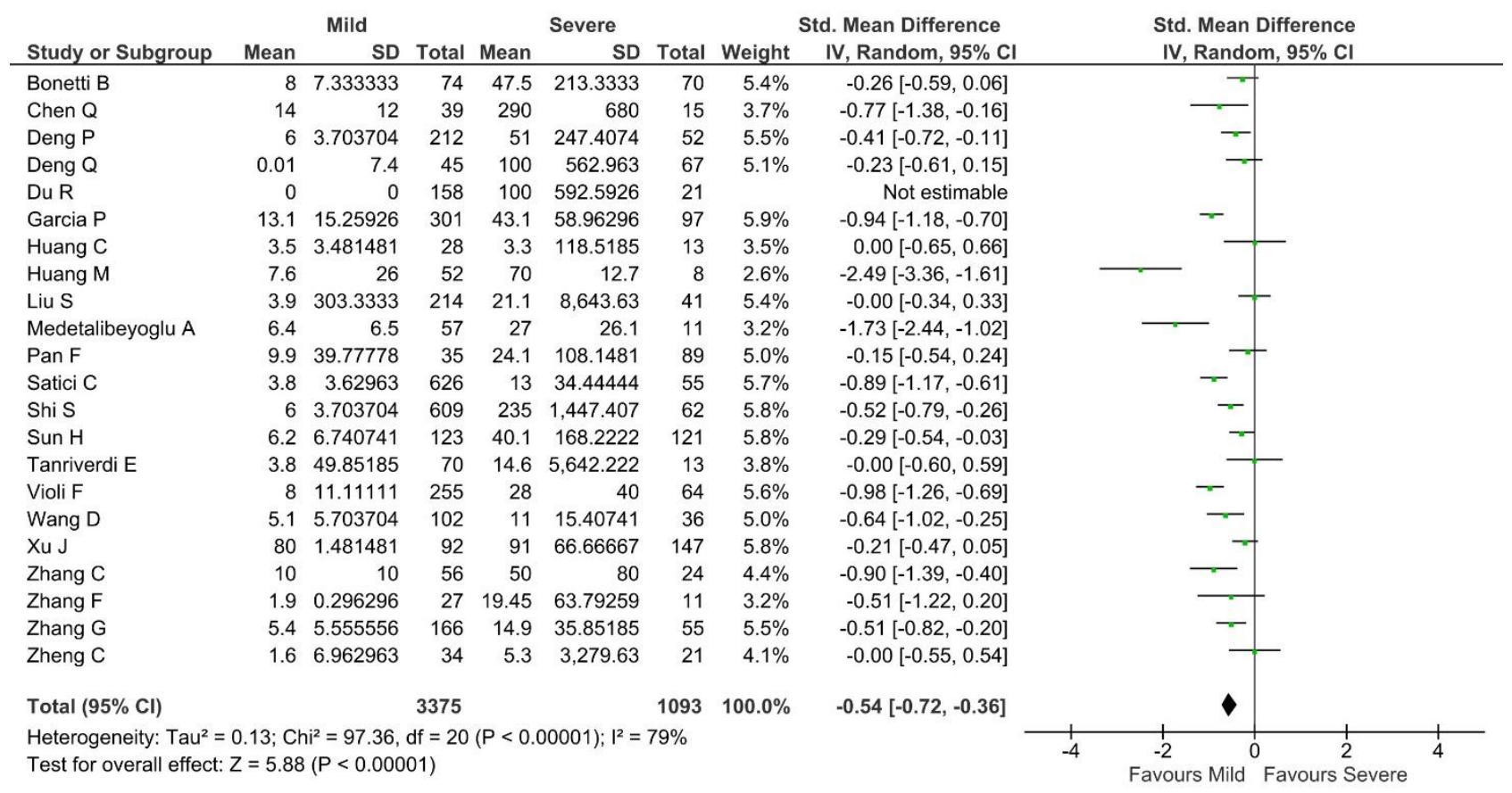

Figure 2. Forest plot of troponin (TnI, ng/mL)

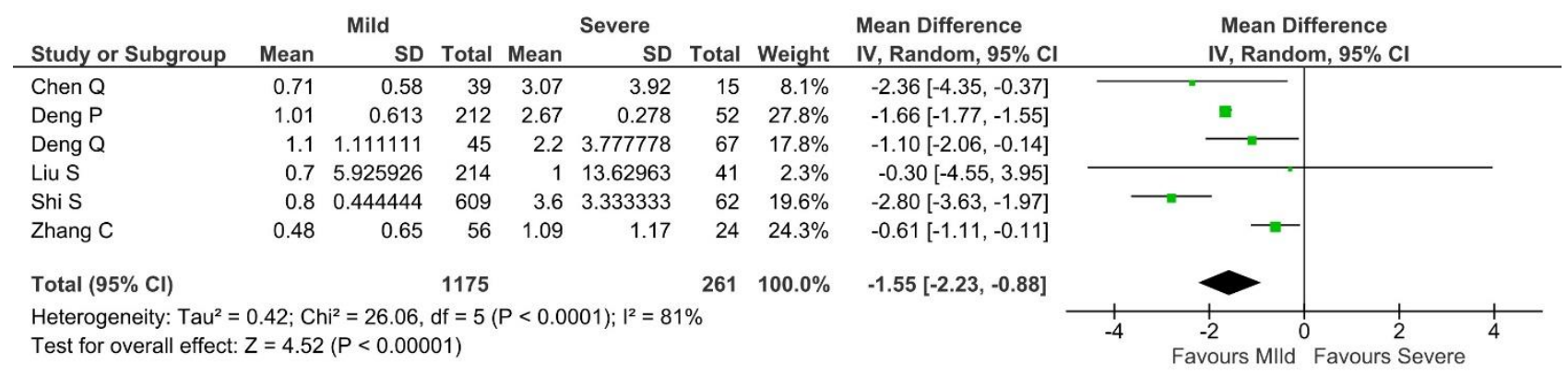

Figure 3. Forest plot of kinase-myocardial band (CK-MB , ng/mL)

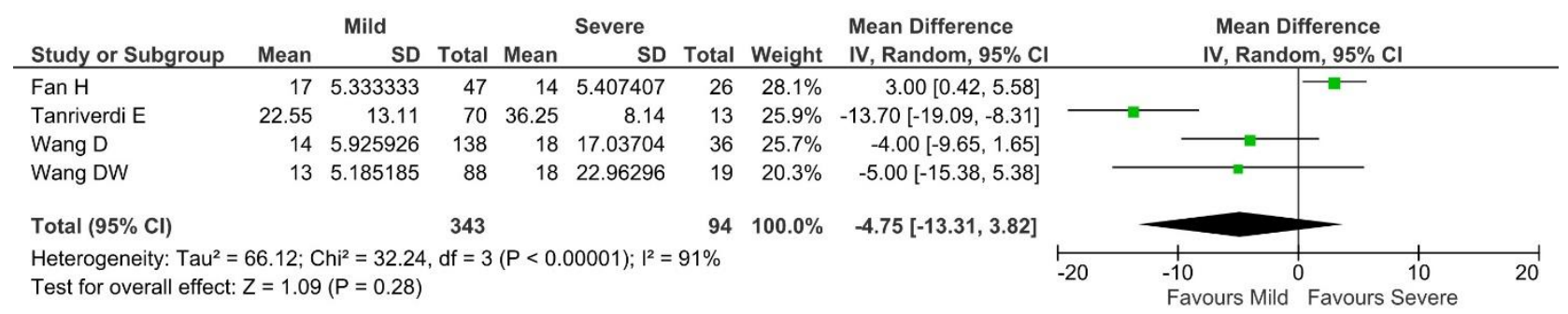

Figure 4. Forest plot of kinase-myocardial band (CK-MB, units/L) 


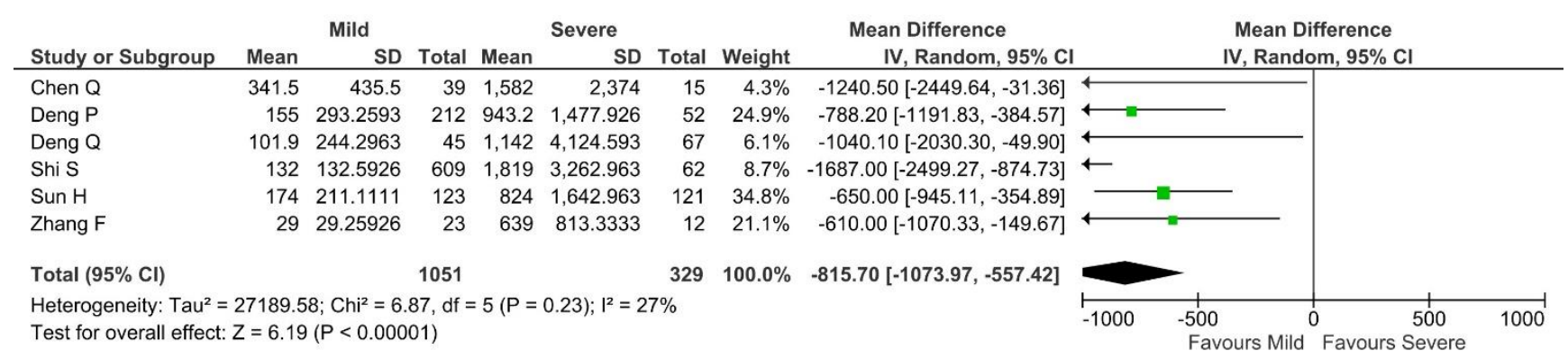

Figure 5. Forest plot of N-terminal-brain natriuretic peptide (NT-BNP, pg/mL)

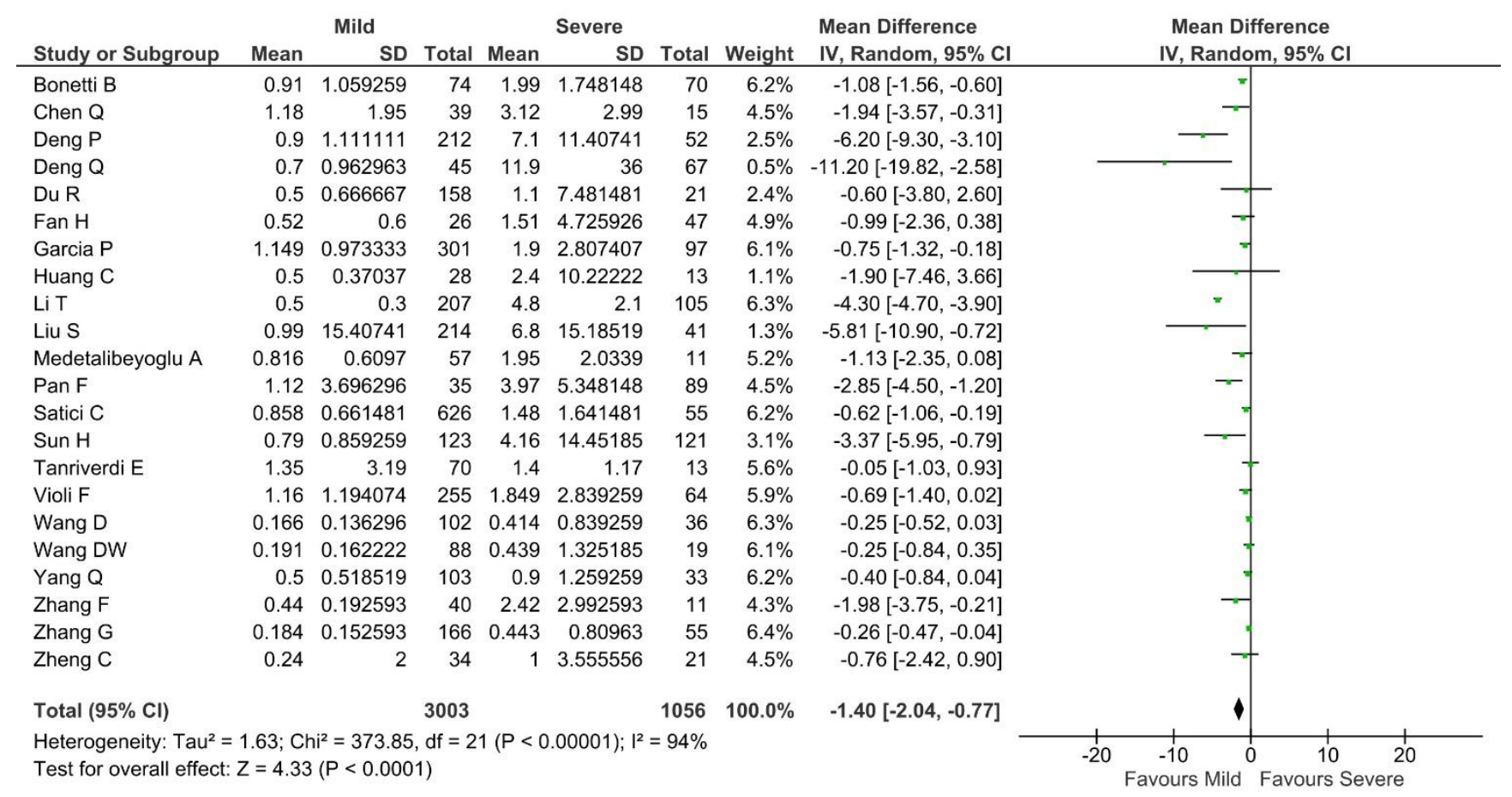

Figure 6. Forest plot of D-dimer $(\mu \mathrm{g} / \mathrm{mL})$

\begin{tabular}{|c|c|c|c|c|c|c|c|c|c|c|c|c|}
\hline \multirow[b]{2}{*}{ Study or Subgroup } & \multicolumn{3}{|c|}{ Mild } & \multicolumn{3}{|c|}{ Severe } & \multicolumn{3}{|c|}{ Mean Difference } & \multirow{2}{*}{\multicolumn{2}{|c|}{$\begin{array}{c}\text { Mean Difference } \\
\text { IV, Random, } 95 \% \mathrm{CI}\end{array}$}} & \\
\hline & Mean & SD & Total & Mean & SD & Total & Weight & IV, Random, $95 \% \mathrm{Cl}$ & & & & \\
\hline Chen $\mathrm{Q}$ & 512 & 350 & 39 & 792 & 608 & 15 & $1.7 \%$ & $-280.00[-606.70,46.70]$ & & & & \\
\hline Deng $\mathrm{P}$ & 282.5 & 116.0741 & 212 & 544 & 282.5926 & 52 & $7.5 \%$ & $-261.50[-339.88,-183.12]$ & & $\longrightarrow$ & & \\
\hline Deng Q & 201 & 74.81481 & 45 & 476 & 315.5556 & 67 & $7.5 \%$ & $-275.00[-353.66,-196.34]$ & & & & \\
\hline Fan $\mathrm{H}$ & 281 & 90.22222 & 26 & 449 & 219.8519 & 47 & $7.8 \%$ & $-168.00[-239.79,-96.21]$ & & & & \\
\hline Garcia P & 465 & 203.7037 & 301 & 506 & 182.2222 & 97 & $8.8 \%$ & $-41.00[-83.95,1.95]$ & & & $\Rightarrow$ & \\
\hline Hong $\mathrm{K}$ & 555.5 & 184 & 85 & $1,272.6$ & 542.1 & 13 & $2.0 \%$ & $-717.10[-1014.37,-419.83]$ & & & & \\
\hline Huang C & 281 & 91.85185 & 28 & 400 & 188.8889 & 13 & $6.4 \%$ & $-119.00[-227.17,-10.83]$ & & & & \\
\hline Li T & 207 & 28.88889 & 19 & 218 & 31.11111 & 15 & $9.3 \%$ & $-11.00[-31.41,9.41]$ & & & & \\
\hline Liu S & 150.4 & 58.4 & 207 & 220.1 & 42.1 & 105 & $9.4 \%$ & $-69.70[-81.02,-58.38]$ & & & " & \\
\hline Medetalibeyoglu A & 273 & 489.1852 & 214 & 509 & 728.1481 & 41 & $2.9 \%$ & $-236.00[-468.32,-3.68]$ & & & & \\
\hline Pan F & 299.4 & 105.6 & 57 & 474.8 & 244.1 & 11 & $5.0 \%$ & $-175.40[-322.23,-28.57]$ & & & & \\
\hline Wang D & 486.1 & 177.3 & 10 & $1,014.3$ & 696.8 & 9 & $0.9 \%$ & $-528.20[-996.51,-59.89]$ & & & & \\
\hline Wang DW & 212 & 88.88889 & 102 & 435 & 217.7778 & 36 & $7.7 \%$ & $-223.00[-296.20,-149.80]$ & & 7 & & \\
\hline Yang Q & 237 & 82.22222 & 9 & 501 & 288.1481 & 39 & $6.5 \%$ & $-264.00[-369.18,-158.82]$ & & & & \\
\hline Zhang G & 251 & 93.33333 & 103 & 398 & 164.4444 & 33 & $8.3 \%$ & $-147.00[-205.93,-88.07]$ & & - & & \\
\hline Zheng C & 204 & 91.11111 & 166 & 424 & 225.1852 & 55 & $8.2 \%$ & $-220.00[-281.10,-158.90]$ & & -7 & & \\
\hline Total $(95 \% \mathrm{Cl})$ & & & 1623 & & & 648 & $100.0 \%$ & $-176.59[-224.11,-129.06]$ & & & & \\
\hline \multicolumn{9}{|c|}{$\begin{array}{l}\text { Heterogeneity: } \text { Tau }^{2}=6200.71 ; \mathrm{Chi}^{2}=176.82, \mathrm{df}=15(P<0.00001) ;\left.\right|^{2}=92 \% \\
\text { Test for overall effect: } Z=7.28(P<0.00001)\end{array}$} & -1000 & $\begin{array}{l}-500 \\
\text { Favours Mild }\end{array}$ & ${ }^{0}$ Favours Severe & $1000^{\prime}$ \\
\hline
\end{tabular}

Figure 7. Forest plot of and lactate dehydrogenase (LDH, units/L) 


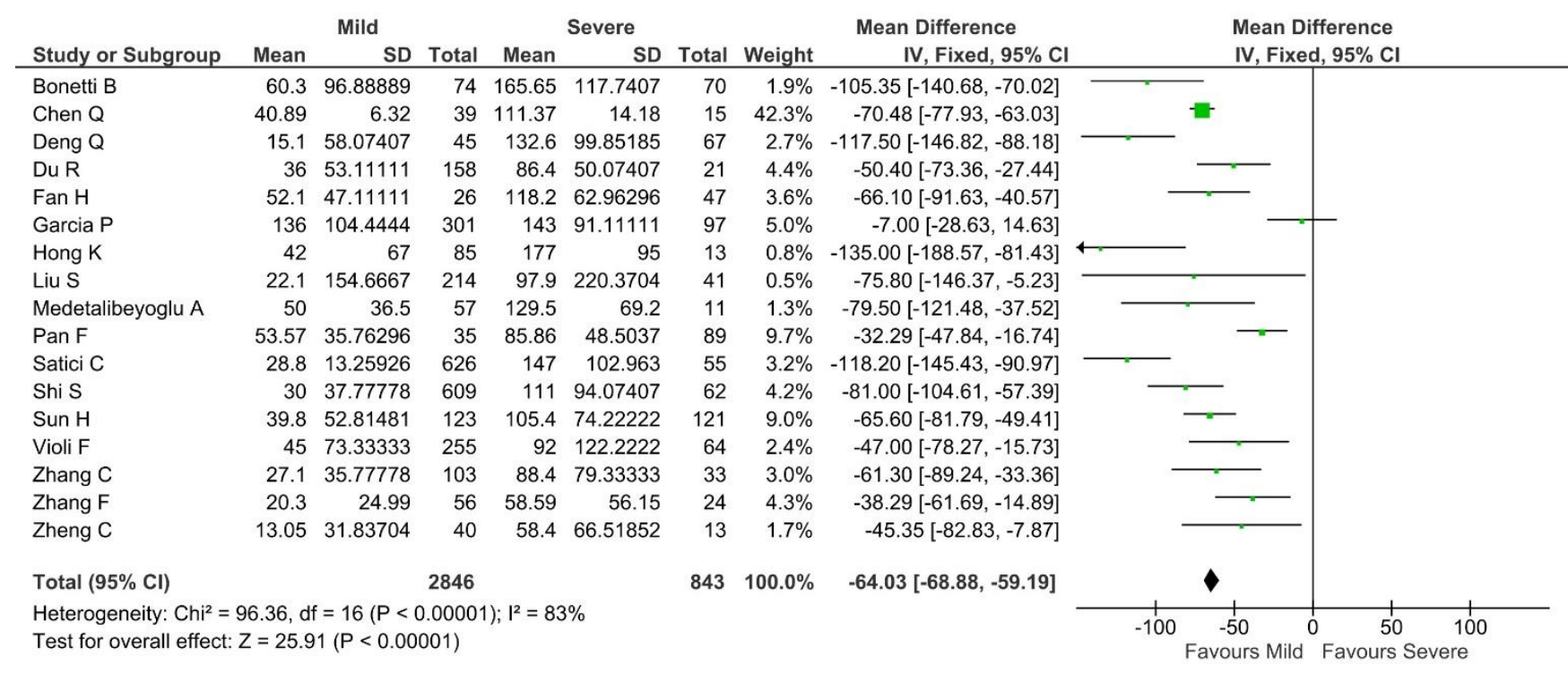

Figure 8. Forest plot of C-reactive protein (CRP, mg/L)

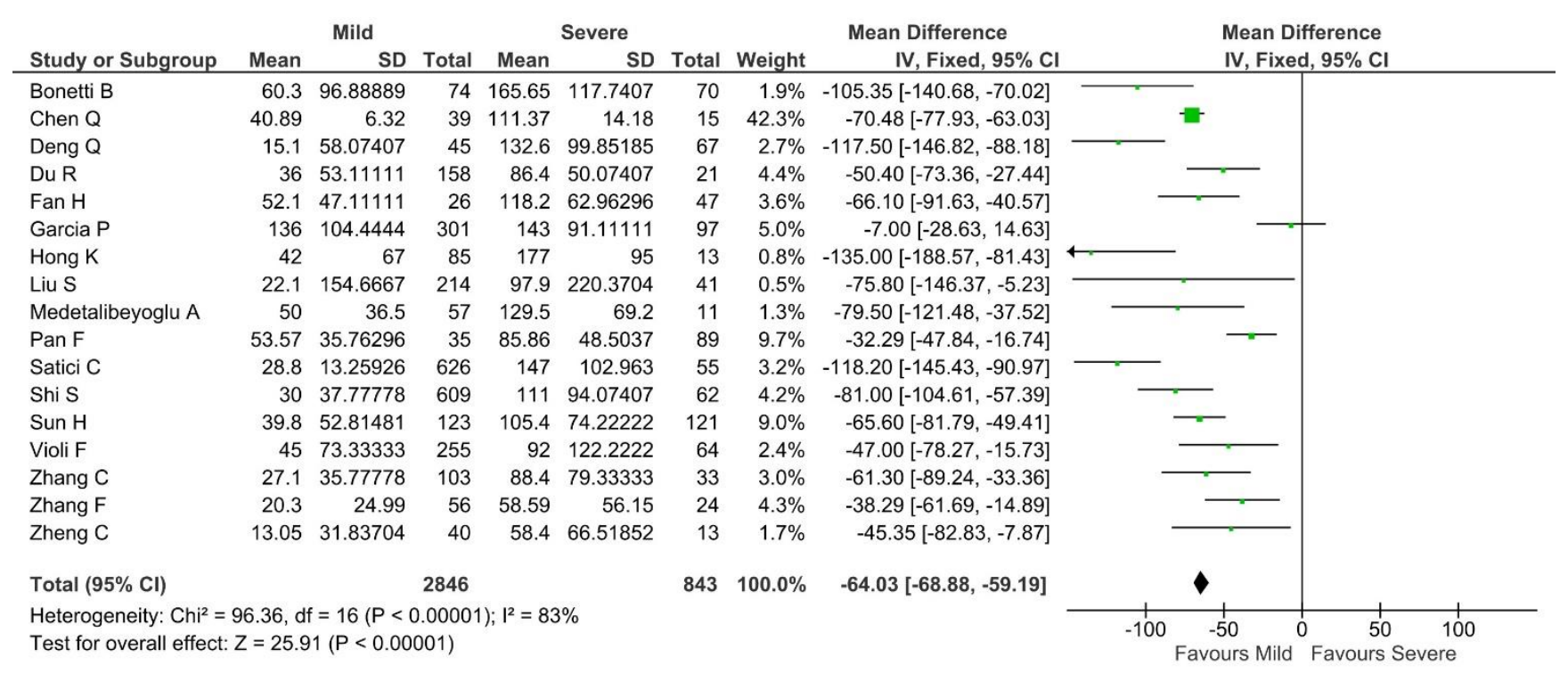

Figure 9. Forest plot of interleukin 6 (IL-6, pg/mL)

\section{REFERENCES}

1. World Health Organization. WHO Director-General's opening remarks at the media briefing on COVID-19 - 11 March 2020. Accessed October 7, 2020..https://www.who.int/dg/speeches/detail/whodirector-general-s-opening-remarks-at-the-mediabriefing-on-covid-19---11-march-2020

2. World Health Organization. Coronavirus disease (COVID19) pandemic. Accessed on October 7, 2020, https://www.who.int/emergencies/diseases/novelcoronavirus2019?gclid=Cj0KCQjw8fr7BRDSARIsAK0Qqr6sMfmiyx790BkvBFn6rg9ManE11wD3syVesvVi_Swr2EHn3bQz YaAiYZEALw_wcB

3. Siripanthong B, Nazarian S, Muser D, et al. Recognizing COVID-19-related myocarditis: The possible pathophysiology and proposed guideline for diagnosis and management. Heart Rhythm 2020;17(9):1463-1471. doi: 10.1016/j.hrthm.2020.05.001. 
4. Gheblawi M, Wang K, Viveiros A, et al. Angiotensinconverting enzyme 2 : SARS-CoV-2 receptor and regulator of the renin-angiotensin system. Circ Res. 2020;126(10):1457-75.

5. Cheng R, Leedy D. COVID-19 and acute myocardial injury: the heart of the matter or an innocent bystander? [Editorial]. Heart 2020; 106(15): 1122-1124. doi: 10.1136/heartjnl-2020-317025.

6. Kindermann I, Barth C, Mahfoud F, et al. Update on Myocarditis. J Am Coll Cardiol. 2012;59:779-792. doi:10.1016/j.jacc.2011.09.074.

7. Elamm C, Fairweather D, Cooper LT. Pathogenesis and diagnosis of myocarditis. Heart. 2012;98(11):835-840. doi: 10.1136/heartjnl-2012-301686.

8. Cooper LT. Myocarditis: Medical Progress. N Engl J Med 2009;360(15):1526-1538. doi: 10.1056/NEJMra0800028.

9. Panic N, Leoncini E, de Belvis G, Ricciardi W, et al. Evaluation of the endorsement of the preferred reporting items for systematic reviews and meta-analysis (PRISMA) statement on the quality of published systematic review and meta-analyses. PLoS One. 2013;8(12):e831138-7. doi: 10.1371/journal.pone.0083138.

10. Stroup DF, Berlin BJ, Sipe TA, et al. Meta-analysis of observational studies in epidemiology: a proposal for reporting. Meta-analysis Of Observational Studies in Epidemiology (MOOSE) group. JAMA.

2000;283(15):2008-2012. doi: 10.1001/jama.283.15.2008.

11. Chen Q, Xu L, Dai Y, et al. Cardiovascular manifestations in severe and critical patients with COVID-19. Clin Cardiol. 2020;43(7):796-802. doi: 10.1002/clc.23384.

12. Medetalibeyoglu A, Senkal N, Capar G, et al. Characteristics of the initial patients hospitalized for COVID-19: a single-center report. Turk J Med Sci. 2020;50(5):1436-1439. doi: 10.3906/sag-2004-98.

13. Zheng C, Wang J, Guo H, et al. Risk-adapted treatment strategy for COVID-19 patients. Int $\mathbf{J}$ Infect Dis. 2020;94:74-77. doi: 10.1016/j.ijid.2020.03.047.

14. Deng P, Ke Z, Ying B, et al. The diagnostic and prognostic role of myocardial injury biomarkers in hospitalized patients with COVID-19. Clin Chim Acta. 2020;510:186190. doi: 10.1016/j.cca.2020.07.018.

15. Du RH, Liang LR, Yang CQ, et al. Predictors of mortality for patients with COVID-19 pneumonia caused by SARSCoV-2: a prospective cohort study. Eur Respir J. 2020;55(5):2000524-12. doi: 10.1183/13993003.005242020.

16. Fan $\mathrm{H}$, Zhang L, Huang B, et al. Cardiac injuries in patients with coronavirus disease 2019: Not to be ignored. Int J Infect Dis. 2020;96:294-7. doi: 10.1016/j.ijid.2020.05.024.

17. Garcia PDW, Fumeaux T, Guerci P, et al. Prognostic factors associated with mortality risk and disease progression in 639 critically ill patients with COVID-19 in Europe: Initial report of the international RISC-19-ICU prospective observational cohort. EclinicalMedicine. 2020;25:100449-100460.

doi: 10.1016/j.eclinm.2020.100449.

18. Shi S, Qin M, Cai Y, et al. Characteristics and clinical significance of myocardial injury in patients with severe coronavirus disease 2019. Eur Heart J. 2020;41(22):20702079. doi: 10.1093/eurheartj/ehaa408.

19. Sun H, Nign R, Tao Y, et al. Risk factors for mortality in 244 older adults with COVID-19 in Wuhan, China: a retrospective study. J Am Geriatr Soc. 2020;68(6):E19E23. doi: 10.1111/jgs.16533.

20. Violi F, Cangemi R, Romiti GF, et al. Is albumin predictor of mortality in COVID-19? Antioxid Redox Signal. 2020 Jun 22. Online ahead of print. doi: 10.1089/ars.2020.8142.

21. Wang D, Hu B, Hu C, et al. Clinical characteristics of 138 hospitalized patients with 2019 novel coronavirus-infected in Wuhan, China. JAMA. 2020;323(11):1061-1069. doi: 10.1001/jama.2020.1585.

22. Yang Q, Xie L, Zhang W, et al. Analysis of the clinical characteristics, drug treatments and prognoses of 136 patients with coronavirus disease 2019. J Clin Pharm Ther. 2020;45(4):609-616. doi: 10.1111/jcpt.13170.

23. Zhang C, Qin L, Li K, et al. A novel scoring system for prediction of disease severity in COVID-19. Front Cell Infect Microbiol. 2020;10:318-15. doi: $0.3389 /$ fcimb. 2020.00318 .

24. Zhang G, Hu C, Luo L, et al. Clinical features and shortterm outcomes of 221 patients with COVID-19 in Wuhan, China. J Clin Virol. 2020;127:104264-10272. doi: 10.1016/j.jcv.2020.104364.

25. Satici C, Demirkol MA, Altunok ES, et al. Performance of pneumonia severity index and CURB-65 in predicting 30day mortality in patients with COVID-19. Int J Infect Dis. 2020;98:84-9. doi: 10.1016/j.ijid.2020.06.038.

26. Bonetti G, Manelli F, Patroni A, et al. Laboratory predictors of death from coronavirus disease 2019 (COVID-19) in the area of Valcamonica, Italy. Clin Chem Lab Med. 2020;58(7):1100-1105. doi: 10.1515/cclm2020-0459.

27. Tanriverdi E, Cortuk M, Yildirim BZ, et al. The use of hydroxychloroquine plus azithromycin and early hospital admission are beneficial in COVID-19 patients: Turkey experience with real-life data. Turk J Med Sci. 2020 Jul 19. Online ahead of print. doi: 10.3906/sag-2005-82.

28. Li T, Lu L, Zhang W, et al. Clinical characteristics of 312 hospitalized older patients with COVID-19 in Wuhan, China. Arch Gerontol Geriatr. 2020;91:104185-104192. doi: 10.1016/j.archger.2020.104185.

29. Liu S, Zhang Q, Wang W, et al. Hyperglycemia is a strong predictor of poor prognosis in COVID-19. Diabetes Res Clin Pract. 2020;167:108338-108346. doi: 10.1016/j.diabres.2020.108338.

30. Deng Q, Hu B, Zhang Y, et al. Suspected myocardial injury in patients with COVID-19: evidence from front-line clinical observation in Wuhan, China. Int $\mathrm{J}$ Cardiol. 2020;311:116-121. doi: 10.1016/j.ijcard.2020.03.087.

31. Hong KS, Lee KH, Chung JH, et al. Clinical features and outcomes of 98 patients hospitalized with SARS-CoV-2 infection in Daegu, South Korea: a brief descriptive study. Yonsei Med. 2020;61(5):431-437. doi: 10.3349/ymj.2020.61.5.431.

32. Huang M, Yang Y, Shang F, et al. Clinical characteristics and predictors of disease progression in severe patients with COVID-19 infection in Jiangsu Province, China: a descriptive study. Am J Med Sci. 2020;360(2):120-128. doi: 10.1016/j.amjms.2020.05.038.

33. $\mathrm{Xu}$ J, Yang X, Yang L, et al. Clinical course and predictors of 60-day mortality in 239 critically ill patients with COVID-19: a multicenter retrospective study from Wuhan, China. Crit Care. 2020;24(1):394-405. doi: 10.1186/s13054-020-03098-9. 
34. Li JW, Han TW, Woodward M, et al. The impact of 2019 novel coronavirus on heart injury: a systemic review and meta-analysis. Prog Cardiovasc Dis. 2020;63(4):518-524. doi: 10.1016/j.pcad.2020.04.008.

35. Santoso A, Pranata R, Wibowo A, et al. Cardiac injury is associated with mortality and critically ill pneumonia in COVID-19: a meta-analysis. Am J Emerg Med. 2020;S0735-6757(20)30280. 2020 April 19. Online ahead of print. doi: 10.1016/j.ajem.2020.04.052.

36. Shoar S, Hosseini F Naderan M, et al. Meta-analysis of cardiovascular events and related biomarkers comparing survivors versus non-survivors in patients with COVID19. Am J Cardiol. 2020;S0002-9149(20)30902-4. 2020 Sep 8. Online ahead of print. doi: 10.1016/j.amjcard.2020.08.044.

37. Sandoval Y, Januzzi JL Jr, Jaffe AS. Cardiac Troponin for Assessment of Myocardial Injury in COVID-19: JACC Review Topic of the Week. J Am Coll Cardiol. 2020;76(10):1244-1258. doi: 10.1016/j.jacc.2020.06.068.

38. Shi S, Qin M, Shen B, et al. Association of cardiac injury with mortality in hospitalized patients with COVID-19 in Wuhan, China. JAMA Cardiol. 20205(7):802-810. doi: 10.1001/jamacardio.2020.0950.

39. Guo T, Fan Y, Chen M, et al. Cardiovascular Implications of Fatal Outcomes of Patients With Coronavirus Disease 2019 (COVID-19). JAMA Cardiol. 2020;5(7):811-818. doi: 10.1001/jamacardio.2020.1017.

40. $\mathrm{Li} \mathrm{Y}, \mathrm{Hu} \mathrm{Y}, \mathrm{Yu} \mathrm{J}$, et al. Retrospective analysis of laboratory testing in 54 patients with severe- or criticaltype 2019 novel coronavirus pneumonia. Lab Invest. 2020;100(6):794-800.

41. Asghar W, Aghazadeh-Habashi A, Jamali F. Cardiovascular effect of inflammation and nonsteroidal anti-inflammatory drugs on renin-angiotensin system in experimental arthritis. Inflammopharmacology. 2017 April 7. doi: 10.1007/s10787-017-0344-1. Epub ahead of print.

42. Aghazadeh-Habashi A, Asghar W, Jamali F. Drug-disease interaction: effect of inflammation and nonsteroidal antiinflammatory drugs on cytochrome P450 metabolites of arachidonic acid. J Pharm Sci. 2018;107(2):757-763. doi: 10.1016/j.xphs.2017.09.020.

43. Gao L, Jiang D, Wen XS, et al. Prognostic value of NTproBNP in patients with severe COVID-19. Respir Res. 2020;21(1):83-90. doi: 10.1186/s12931-020-01352-w.

44. Unudurthi SD, Luthra P, Bose RJC, et al. Cardiac inflammation in COVID-19: Lessons from heart failure. Life Sci. 2020;260:118482-118493. doi: 10.1016/j.lfs.2020.118482.

45. Huang C, Wang Y, Li X, et al. Clinical features of patients infected with 2019 novel coronavirus in Wuhan, China. Lancet. 2020;395(10223):497-506. doi: 10.1016/S0140-6736(20)30183-5.

46. Pan F, Yang L, Li Y, et al. Factors associated with death outcome in patients with severe coronavirus disease-19 (COVID-19): a case-control study. Int J Med Sci. 2020;17(9):1281-1292. doi: 10.7150/ijms.46614.

47. Moreau A, Le Vee M, Jouan E, et al. Effect of gevokizumab on interleukin-1beta-mediated cytochrome P450 3A4 and drug transporter repression in cultured human hepatocytes. Eur J Drug Metab Pharmacokinet. 2017;42(5):871-878. doi:10.1007/s13318-017-0406-1.
48. Dhama K, Sharun K, Tiwari R, et al. COVID-19, an emerging coronavirus infection: advances and prospects in designing and developing vaccines, immunotherapeutics, and therapeutics. Hum Vaccin Immunother. 2020;16(6):1232-1238. doi:10.1080/21645515.2020.1735227.

49. Bernardini A, Ciconte G, Negro G, et al. Assessing QT interval in COVID-19 patients: safety of hydroxychloroquine-azithromycin combination regimen. Int J Cardiol. 2020;S0167-5273(20:33819-33824. doi: 10.1016/j.ijcard.2020.09.038.

50. Cavalcanti AB, Zampieri FG, Rosa RG, et al. Hydroxychloroquine with or without azithromycin in mild-to-moderate Covid-19. N Engl J Med. 2020 Jul 23. doi: 10.1056/NEJMoa2019014. Online ahead of print.

51. Hsia BC, Greige N, Quiroz JA, et al. QT prolongation in a diverse, urban population of COVID-19 patients treated with hydroxychloroquine, chloroquine, or azithromycin. J Interv Card Electrophysiol. 2020 Jul 11;1-9. doi: 10.1007/s10840-020-00822-x. Online ahead of print.

52. Maraj I, Hummel JP, Taoutel R, et al. Incidence and determinants of QT interval prolongation in COVID-19 patients treated with hydroxychloroquine and azithromycin. 2020 Jun 2. doi: 10.1111/jce.14594. Online ahead of print.

53. The U.S. Food \& Drug Administration. FDA drug safety communication. FDA cautions against use of hydroxychloroquine or chloroquine for COVID-19 outside of the hospital setting or a clinical trial due to risk of heart rhythm problems. Does not affect FDA-approved uses for malaria, lupus, and rheumatoid arthritis. Safety Announcement [04-24-2020]. Accessed on Oct 14, 2020. https://www.fda.gov/media/137250/download

54. Kohan H, Jamali F. Anti-inflammatory properties of drugs used to control COVID-19 and their effects on the reninangiotensin system and angiotensin-converting enzyme- 2 . J Pharm Pharm Sci. 2020;23:259-277. doi: 10.18433/jpps31346. 